\title{
ON THE SYMBOL OF A PSEUDO-DIFFERENTIAL OPERATOR
}

\author{
BY WEISHU SHIH ${ }^{1}$
}

Communicated by Richard S. Palais, January 17, 1968

In [1] Hörmander defines the generalized symbol of a pseudodifferential operator $P$ as a sequence of partially defined maps between function spaces. Our purpose here is to comment on the existence of characteristic polynomial type symbols $\sigma(P)$ and to obtain their composition by introducing a product structure on suitable jet bundles. In particular, this gives the lower order symbol for differential operator on manifold. I express my hearty thanks to J. Bokobza, $H$. Levine, and A. Unterberger for their indispensable help.

1. Operation in jet bundle. Given a compact $C^{\infty}$ differentiable manifold $X$, we denote by

$$
p_{k}: J^{m}(R) \rightarrow J^{k}(R), \quad m \geqq k,
$$

the jet bundle of the trivial bundle $X \times R$ and the canonical projection. Identify the cotangent bundle $T(X)$ as a subbundle of $J^{1}(R)$ we define the subbundle

$$
J_{0}^{k}(R) \subseteq J^{k}(R), \quad k \geqq 1,
$$

as the inverse image by $p_{1}: J^{k}(R) \rightarrow J^{1}(R)$ of the nonzero cotangent vector $T_{0}(X) \subseteq T(X)$. Let $E, F$, and $G$ be complex vector bundles over $X$ and put

$$
J^{*}(E, F)=\prod_{k=0} \operatorname{Hom}\left(J_{0}^{k+1}(R) \oplus J^{k}(E), F\right)
$$

where "Hom" denotes the space of $C^{\infty}$ bundle maps which are linear with respect to $J^{k}(E)$. We shall construct an operation

$$
\circ: J^{*}(E, F) \times J^{*}(F, G) \rightarrow J^{*}(E, G)
$$

as follows. If $\alpha=\left(\alpha_{0}, \alpha_{1}, \cdots, \alpha_{m}, \cdots\right) \in J^{*}(E, F), \beta=\left(\beta_{0}, \beta_{1}, \cdots\right)$ $\in J^{*}(F, G)$, then

$$
\alpha \circ \beta=\left(\gamma_{0}, \gamma_{1}, \cdots, \gamma_{r}, \cdots\right) \in J^{*}(E, G)
$$

is given by

$$
\gamma_{r}=\sum_{m+n=r} \beta_{n} \circ\left(p_{n+1} \circ p_{R} \oplus j^{n}\left(\alpha_{m}\right)\right)
$$

\footnotetext{
${ }^{1}$ Research supported by National Science Foundation Grant GP5804.
} 
where $p_{R}: J_{0}^{*}(R) \oplus J^{*}(E) \rightarrow J^{*}(R)$ is the projection, and

$$
j^{n}: \operatorname{Hom}\left(J_{0}^{k+1}(R) \oplus J^{k}(E), F\right) \rightarrow \operatorname{Hom}\left(J_{0}^{k+n+1}(R) \oplus J^{k+n}(E), J^{n}(F)\right)
$$

the $n$th jet extension map.

THEOREM. The operation "o" is well defined, associative, and distributive. Moreover if the $\alpha_{m}$ (resp. $\beta_{n}$ ) in $\alpha$ (resp. $\beta$ ) is positive homogeneous of degree $k-m$ (resp. $h-n)$ with respect to $J_{0}^{m+1}(R)$, then $(\alpha \circ \beta)_{r}$ is positive homogeneous of degree $k+h-r$ ( $k, h$ real numbers). In particular, with respect to this operation $J^{*}(E, E)$ becomes an associative algebra with unity [2].

2. The symbol homomorphism. Let us recall that a continuous linear map

$$
P: C^{\infty}(E) \rightarrow C^{\infty}(F)
$$

between the space of $C^{\infty}$ sections of complex vector bundles is a pseudo-differential operator of order $k$ in the sense of Hörmander if: for each $f \in C^{\infty}(E), g \in C^{\infty}(R)$, such that

$$
\text { supp } f \subseteq \operatorname{supp}^{0} d g \text {; interior of support of } d g
$$

there is a uniform asymptotic expansion [1]

$$
e^{-i \lambda g} P\left(e^{i \lambda \sigma} f\right) \sim \sum_{0}^{\infty} P_{j}(g, f) \lambda^{k-j}
$$

with $P_{j}(g, f) \in C^{\infty}(F)$ and $P_{0}(g, f) \neq 0$. The formal sum $\sum_{0}^{\infty} P_{j}(g, f)$ is the generalized symbol of $P$.

Now let us denote by

$$
\odot(E, F)=\sum_{k} \odot_{k}(E, F)
$$

the space of all pseudo-differential operators from the complex vector bundle $E$ to the bundle $F$ over the fixed compact manifold $X ; P_{k}(E, F)$ those of order $k$. Then we have

THEOREM. There exists a unique homomorphism

$$
\sigma: \odot(E, F) \rightarrow J^{*}(E, F)
$$

satisfying the following conditions:

(1) If $P \in \mathcal{P}_{k}(E, F)$, then $\sigma_{j}(P)$ is positive homogeneous of degree $k-j$ with respect to $J_{0}^{j+1}(R)$ where $\sigma(P)=\left(\sigma_{0}(P), \sigma_{1}(P), \cdots\right)$.

(2) If $P \in \mathcal{P}(E, F), Q \in \mathcal{P}(F, G)$, then

$$
\sigma(P \circ Q)=\sigma(P) \circ \sigma(Q) \text {. }
$$


(3) If $P \in \rho_{k}(E, F)$ and $f \in C^{\infty}(E), g \in C^{\infty}(R)$, verify the condition $\left.{ }^{*}\right)$, then the generalized symbol of Hörmander $P_{j}(g, f)$ is equal to the image of $(d g, f)$ by the composition

$$
C^{\infty}\left(T_{0}(X)\right) \times C^{\infty}(E) \rightarrow C^{\infty}\left(J_{0}^{j+1}(R) \oplus J^{j}(E)\right) \stackrel{\sigma_{j}(P)}{\longrightarrow} C^{\infty}(F)
$$

restricted on the interior of the support of $d g$.

(4) If $P$ is a kth order differentiable operator, then $\sigma_{j}(P)$ is defined on $J^{j+1}(R) \oplus J^{j}(E)$ and is zero for $j>k$. Moreover the restriction of $\sigma_{0}(P)$ on $T_{0}(X) \subseteq J^{1}(R)$ :

$$
\sigma_{0}(P): T_{0}(X) \oplus E \rightarrow F
$$

is the classical [3] symbol of the differential operator $P$.

REMARK: $\sigma: \rho(E, E) \rightarrow J^{*}(E, E)$ is a homomorphism of algebra with unity. Choose a splitting (e.g. by connections). We obtain an inclusion $T_{0}(X) \oplus E \hookrightarrow J_{0}^{j+1}(R) \oplus J^{j}(E)$; then the restriction of $\sigma_{j}(P)$ on $T_{0}(X) \oplus E$ gives the lower order characteristic polynomial of $P$ (e.g. in the case of $R^{n}$ one gets back the ordinary total characteristic polynomial of a differential operator). Using jet bundles [5] along the fiber, one obtains the same result for a family of operators.

\section{REFERENCES}

1. L. Hörmander, Pseudo-differential operator, Comm. Pure Appl. Math. 18 (1965), 501-517.

2. J. J. Kohn and L. Nirenberg, An algebra of pseudo-differential operators, Comm. Pure Appl. Math. 18 (1965), 269-305.

3. R. Palais, Lecture notes, Brandeis, Univ., Waltham, Mass., 1964.

4. R. T. Seeley, Singular integrals on compact manifolds, Amer. J. Math. 81 (1959), 658-690.

5. Weishu Shih, Fiber cobordism and the index of a family of elliptic differential operators, Bull. Amer. Math. Soc. 72 (1966), 984-991.

6. A. Unterberger and J. Bokobza, Les operators de Calderon-Zygmund precises, C. R. Acad. Sci. Paris 259 (1964), 1612-1614.

INSTITUTE For Advanced STUdy AND

Institute Hautes Etudes Scientifiques 